$30 \mid 2003$

Les Aventures de Télémaque. Trois siècles

d'enseignement du français. I.

\title{
L'approcio non-autoritario nelle pedagogia di Fénelon
}

\section{Carlo Pancera}

\section{(2) OpenEdition \\ Journals}

Édition électronique

URL : https://journals.openedition.org/dhfles/1520

DOI : $10.4000 /$ dhfles. 1520

ISSN : 2221-4038

Éditeur

Société Internationale pour l'Histoire du Français Langue Étrangère ou Seconde

Édition imprimée

Date de publication : 1 juin 2003

ISSN : 0992-7654

Référence électronique

Carlo Pancera, «L'approcio non-autoritario nelle pedagogia di Fénelon », Documents pour l'histoire du français langue étrangère ou seconde [En ligne], 30 | 2003, mis en ligne le 01 janvier 2012, consulté le 27 mai 2021. URL : http://journals.openedition.org/dhfles/1520 ; DOI : https://doi.org/10.4000/dhfles. 1520

Ce document a été généré automatiquement le 27 mai 2021

(c) SIHFLES 


\title{
L'approcio non-autoritario nelle pedagogia di Fénelon
}

\author{
Carlo Pancera
}

1 La concezione dell'educazione generalmente condivisa all'epoca di Fénelon era tesa a fini morali : sono questi che conferiscono un senso alla stessa opera di formazione delle nuove generazioni ; riflettere sulle modalità dell'intervento formativo è, in un momento in cui ci si interroga sui valori correnti e sulle consuetudini sociali, di rilevanza essenziale. La pedagogia sino ad allora ha sempre avuto come primario suo scopo la formazione morale e spirituale della nuova generazione : tutto ciò che si ritiene degno di essere insegnato è tale per il fatto che veicola determinati valori, concorre alla elevazione spirituale e all'affinamento della persona. L'educazione è ritenuta un'arte inerente al perfezionamento morale della civiltà. Si afferma in questo periodo, almeno nella storia della letteratura pedagogica, il genere trattatistico. Ma Fénelon, in pedagogia, è un anti-sistematico, è contrario all'esprit de géométrie, alla elaborazione di un compatto modelo teorico che faccia da supporto ad una serie rigorosa di derivati.

2 Fénelon non è un autore di un trattato in cui esporre le proprie convinzioni, almeno in materia pedagogica o didattica (anche se in parte ricorre in un certo senso a questo genere nell'opera De l'existence et des attributs de Dieu, non a caso generalmente nota come Démonstration). Nemmeno quello che diverrà noto con la denominazione di " Traité de l'éducation des filles " (in realtà da lui intitolato semplicemente De l'éducation des filles) è strutturato come una esposizione organica e ragionata di un sistema pedagogico (come fecero ad esempio Rollin, o padre Jouvancy con la sua De ratione docendi et discendi). Fénelon deliberatamente non teorizza, né fissa princìi in un testo di carattere filosofico teorético sulla educazione, sia essa des filles che del principe, ma piuttosto articola in una serie di esempi e di indicazioni, da commisurare poi ai singoli allievi considerati nella loro specificità individuale. In quasi tutti i suoi scritti risulta evidente l'interesse per l'educazione e per le sue problematiche. Le tipologie feneloniane non distinguono per rango (e nemmeno sempre per sesso), come era d'uso, bensì per tipi psicologici : l'apatico, il simulatore, l'egocentrico, l'affettuoso... Proprio 
nel suo testo sull'educazione delle fanciulle c'è questo interessantissimo esame di tipi psicologici. Si tratta in effetti, qui come altrove, di consigli intesi come tessere di mosaico che potrebbero contribuire a comporre una sorta di filosofía pratica della vita, e che si potrebbero anche leggere a parte come proposte sui comportamenti da tenersi con i vari tipi di individui. È nel campo psicologico, e conseguentemente in quello delle modalità dell'apprendimento, che dunque egli ritiene vi siano caratteristiche generali che attraversano gli strati sociali, identificabili negli esseri umani nel loro insieme.

Innanzitutto, come accennerò più avanti, vi sono per lui anche varie specie di razionalità, ma comunque la ragione nel suo complesso non è ciò che rende l'uomo un essere superiore, $\mathrm{o}$ - ed è più importante - ciò che lo può salvare, ma è semmai la fede.

Quindi è essenziale per Fénelon iniziare i giovani alla spiritualità, coltivarla in loro, ed elevare l'allievo gradualmente alla più alta spiritualità che sia acquisibile per ciascuno. Per giungere infine, per quanto possibile, a desiderare le don de soi. Ma ciò si inscrive in un non agevole e lungo percorso. È la sua théologie du sacrifice. La virtù suprema cui formare gli animi è quella di compiere sacrificio di sé per fare il bene ${ }^{1}$. Fénelon dunque non va inteso tout-court come un fautore della tolleranza in senso ampio, se non nell'accezione dell'utilizzo di una pedagogía dolce nell'approccio all'altro, al catechizzando, all'educando, all'allievo. Così come la sua propensione ad una pedagogia, e ad una politica non autoritaria è da considerarsi piuttosto come un atteggiamento che si muove in sintonia con la reazione nobiliare anti-assolutista. Tutti i suoi scritti, e in generale tutta la sua opera politica, morale, religiosa e pedagogica formano in modo coerente un único grande progetto riformatore che inizia con il testo sull'educazione delle fanciulle, a cui succedono interventi che vengono nel corso del tempo strutturando, elaborando e precisando un ampio mosaico di notevole fattura e bellezza, un modello assai complesso, ma organico, di riforma sociale e spirituale.

Le sue concezioni dell'educazione sono anche frutto di dibattiti collettivi, di confronti dialettici, di dialoghi con uomini e donne di cultura e di fede, e di un continuo confronto e verifica con l'esperienza concreta. Fondamentale tra i molti è il suo rapporto di amicizia fraterna con Fleury.

6 Il recupero dei grandi classici è fondamentale, non tanto per partito preso nella Querelle des Anciens et des Modernes, ma in vista di una loro rivisitazione e reinterpretazione del loro umanesimo. Chiara è l'approche humaniste di Fénelon ai temi politici, educativi e morali. La sua rilettura dei classici è compiuta comunque sempre ai fini di un arricchimento della concezione cristiano-cattolica della vita. È poco congruente ad esempio l'accostamento a volte fatto da storici con Montaigne. Così come il suo amore per i classici è da vedersi come una rilettura dei valori umanistici espressi nella storia dell'occidente, e soprattutto una valorizzazione del senso storico, che ritiene di grande importanza coltivare nei giovani. Si vedano le sue Réflexions sur la grammaire, la rhétorique, la poétique et l'histoire, i Dialogues sur l'éloquence, la Lettre à l'Académie. Si tratta inoltre di riempire di contenuti, anche con il ricorso al pensiero dei classici, il concetto di honnête et habile homme in chiave cristiana. Il principe stesso, come tutti, ma a maggior ragione, deve essere " esperto della vita ", modalità indispensabile per sostanziare di esperienze un percorso di formazione. Percorso ovviamente indirizzato e pilotato dalla guida, dal mentore.

7 In pedagogia, ma non solo, Fénelon non è autore che faccia affidamento sulla cosiddetta " spontaneità infantile ». Nel Télémaque (cf. Libro IV) si mostra come anche nelle età infantile e adolescenziale le passioni più genuine possano divenire quantomeno 
sospette se non sottoposte a costante esame critico. In effetti percorre tutto il suo pensiero, religioso, filosofico, letterario, una generale riflessione sulla natura umana, riflessione che rende conto delle possibilità stesse della pedagogia ; e in ciò Fénelon non è certo, come sovente si dice, un antesignano delle concezioni rousseauiane, poiché non crede l'uomo naturalmente buono.

In queste riflessioni possiamo identificare la sua idea di ragione. Fénelon non è certamente un razionalista secentesco, ma nemmeno un precursore delle Lumières. Senza dubbio, a suo avviso bisogna saper perfezionare l'uso della ragione. Ma la ragione non è solo l'intelletto che analizza, calcola e compie astrazioni. Fénelon non accorda completa fiducia alle risorse della razionalità logica. D'altronde basta osservare le differenze che rileva tra maschio e femmina. Se l'uno per natura è più propenso ad un tipo di razionalità teoretica e speculativa, nell'altra si può osservare (e in questo concordavano con lui sia Madame de Maintenon, che Madame Guyon) una razionalità più pratica e concreta, e meno propensa all'astrazione. Una concezione della ragione umana dunque intesa solo come abilità logica, non sarebbe in accordo con la sua visione intuitiva della conoscenza, la quale non è qualcosa di intessuto di categorie e definizioni o classificazioni.

La ragione e la psicologia degli individui sono difficilmente scindibili, e Fénelon sembra comunque dare all'analisi della seconda la precedenza. La riflessione sulla natura umana necessita a sua volta di andare oltre il dato psicologico, il quale riguarda comunque soprattutto le modalità di conoscenza, il peculiare tipo di approccio alla realtà e alle relazioni sociali di ogni singola personalità ; mentre la riflessione in generale sulla natura umana riguarda piuttosto le possibilità stesse, e i limiti, della conoscenza che l'uomo può avere del significato delle cose del mondo. Sono le modalità di apprendimento che ci possono spiegare come si formi la personalità di ogni essere umano. Inoltre l'aver chiaro cosa sia il proceso di apprendimento nella natura umana è un dato fondamentale per poi poter procedere nell'illustrazione di ciò che rende possibile un intervento pedagogico $: \mathrm{i}$ suoi prerequisiti. Utilizzando una metafora della letteratura classica, accosta l'apprendimento più che ad un processo di analisi e di astrazioni, alla digestione dei cibi (già Socrate utilizzava una simile metafora, secondo quanto ci riferisce Platone, di cui l'amico Fleury era studioso).

Fénelon cerca di farsi intendere ricorrendo alla metafora che consente di cogliere in modo immediato, lasciando così un segno più profondo nello spirito. Si veda, ad esempio, la metafora del ruscello referente della ragione umana, ruscello, magari minuscolo, ma sufficiente per l'uso che ne dobbiamo fare (cf. De l'existence de Dieu). Piuttosto che pretendere un'autosufficienza o anche solo una autonomia, la ragione come appunto un ruscello, non deve di fatto far altro che raccordarsi a quella luce semplice, infinita, immutabile, che si manifesta nella Parole de Dieu, nel Verbo. Essa sola è veritativa, essa è come un grande oceano, che solo la pratica della meditazione ci svela e a cui ci rende sensibili : al punto da desiderare quasi di perdervisi, come un ruscello in un oceano fascinoso (cf. Télémaque, IV). È a queste immagini che Fénelon ricorre.

11 Ad esempio, in De l'existence de Dieu, scrive che noi riceviamo i Lumi dalla Ragione Suprema ad ogni istante della nostra vita, così come respiriamo l'aria. Sono suggestioni che forse gli derivano dal modo di esprimersi di Madame Guyon, o forse da letture del tardoplatonismo cristiano. La ragione umana in generale è una sorta di coscienza intima della Ragione Suprema che viene risvegliata in noi dalla frequente pratica di 
esercizi spirituali e in primo luogo dalla meditazione sulle sacre scritture (Gouhier 1977). È evidente il raccordo con la pedagogia poiché il pericolo che la guida spirituale, o il directeur de conscience, sostituisca la propria ragione a quella dell'allievo. Il che poi sarebbe possibile solo imponendo, e quindi sostituendo, la propria volontà a quella dell'educando, e giustificando, o facendo sì che l'allievo giunga a giustificare lui stesso questa operazione, di cui non è pienamente consapevole nella sua coscienza, con la ragione. È ciò che chiameremmo plagio, abuso così frequente secondo Fénelon nella pratica pedagogica corrente ai suoi tempi negli istituti educativi religiosi (cf. anche la critica alla formazione data dalle suore in De l'éducation des filles). Come dirà Foucault, il confessore parla ed ecco enunciato il potere della regola, per cui si innesca un ciclo perverso di ciò che è interdetto, che porta ad interiorizzare la logica censoria come pratica di autocensura preventiva. Il penitente dunque non riesce più a vivere né a ragionare apertamente : « tu n'existeras pas, sauf dans l'ombre » (Foucault $1976: 110$ ). Ma per Fénelon la ragione è una delle vie di conoscenza che possediamo e ogni individuo ha i suoi peculiari modi e percorsi per accedere alla conoscenza. Se così non fosse, la pedagogia (come anche la retorica, v. Lettre à l'Académie) diverrebbe null'altro che manipolazione, o per lo meno, arte del condizionamento operata oltretutto senza che l'allievo capisca verso quale obiettivo lo si sta orientando né i metodi che si stanno utilizzando. Accadrà così anche ad Emilio con la « ruse » del suo astuto precettore alias Rousseau. Fénelon non apprezza queste finezze della ragion pedagogica; preferisce « insegnare senza averne l'aria », di classica e umanistica reminiscenza (ma non si equivochi pensando per questo ad un Fénelon ispirato solamente dal buon senso o dalla condiscendenza... !).

La precettistica pedagogica, confusa con la pedagogia in quanto tale (ecco un altro motivo del rifiuto del genere trattatistico) si poteva applicare a qualsivoglia caso, così come la metodologia didattica, e spesso si partiva dalla Teoria anche solo per conferire una caratteristica identitaria alla propria scuola (gli Oratoriani, i Gesuiti...). Non così Fénelon. Egli adotta sempre un approccio in qualche modo individualizzato, partendo da osservazioni psicologiche, senza tempi rigorosi da rispettare e facendo soprattutto leva sulle motivazioni. Anche qui gli si attaglia il motto " prender tempo per guadagnarlo ", altro topos classico e umanistico.

Nel suo ottimismo Fénelon ha sì fiducia nelle capacità della ragione, ma ancor più nella forza di spirito dell'allievo di cui coltiva in primo luogo la sensibilità e la spiritualità che si riflettono nel mondo della natura. C'è sovente in Fénelon questo gioco di rispecchiamenti : anche la natura è uno specchio ${ }^{2}$.

Negli autori di letteratura pedagogica di questa epoca ci troviamo di fronte a due possibili interpretazioni (cf. Jolibert 1977) : l'una che potremmo indicare con le naturel en nous. La nostra natura è così indeterminata che l'allievo potrebbe divenire quasi qualsiasi tipo di persona, dall'enfant si può giungere a fare l'adulto che si vuole. L'educazione può dunque essere un atto forgiativo di grande potere : siamo in realtà il frutto dell'educazione subita, che ci ha plasmato. Potremmo chiamare : la détermination première la seconda possibile interpretazione. Ciò che è naturalmente predeterminato in ciascuno di noi, relativamente alle nostre individuali caratteristiche e inclinazioni, lo è così nettamente che l'educazione può influire ben poco sullo sviluppo della nostra personalità. L'educazione intellettuale e del comportamento in effetti non sarebbe che una sorta di tintura esteriore, poco più che un abito, che mostriamo in pubblico. L'opera di formazione non sarebbe dunque che l'arte di estrapolare ciò che è già dato. 

meglio egli non va letto attraverso queste categorie che possono essere utili e chiarificatrici del pensiero pedagogico post-feneloniano. Per Fénelon va in primo luogo distinto, decifrato, il carattere individuale, che va conosciuto e compreso nella fase iniziale, diremmo preliminare allo svolgersi dell'opera educativa : poiché per Fénelon è il gouverneur che innanzitutto deve piegarsi, adattarsi a tale carattere per perfezionarlo e volgerlo a dare i suoi frutti migliori. Possiamo affermare che secondo Fénelon non è concesso fare ciò che si vuole degli esseri umani. Questo è per lui un precetto fondamentale in pedagogia, in filosofia e in politica. Poiché ritiene che molto si possa influenzare e condizionare un fanciullo, l'opera educativa è assai delicata e va affrontata con perizia e con tatto. suoi tempi, termineremo con una delusione. Fénelon non crede né che l'anima umana sia stata irrimediabilmente pervertita dal peccato originale, né che essa sia naturalmente buona quando segue la propria spontaneità ${ }^{3}$.

per questo Fénelon ritiene fondamentale la tolleranza con gli esseri umani, poiché forzarli a ciò che non sono propensi a fare è un'azione dalle conseguenze controproducenti. D'altro canto lasciare il fanciullo libero in tutto e per tutto, è certo errato. Bisogna aiutare l'individuo a correggersi da sé, a volere perfezionare le proprie stesse capacità. In questa delicata operazione, che richiede tempo, pazienza, continuità e quindi determinazione, l'educatore deve saper essere riservato, aver rispetto per la personalità dell'allievo, e adattarsi alla sua modalità di esprimersi, alle sue possibilità e ai suoi limiti. L'allievo dev'essere corretto con dolcezza e pazienza, senza mai fare ricorso a punizioni che segnalerebbero uno scacco per l'educatore, una ammissione di debolezza che guasterebbe il rapporto stesso dell'educatore con l'allievo, avviandoli ad un percorso contorto e denso di tensioni, da cui sarebbe difficile tornare indietro. Il gouverneur dovrà « limitarsi » a gettare dei semi nell'animo dell'allievo. Semi che prima o poi germineranno, semi scelti oculatamente, che siano suscettibili di sviluppo con le esperienze che il giovane compirà, e che divengano per lui punti di riferimento, o per lo meno oggetti di rielaborazione.

Fénelon è pure distante dalla tradizionale pedagogia religiosa della Imitatio Christi che non propone ai suoi allievi. Certo la vita di Gesù ci mostra la strada da seguire, i Vangeli sono pieni di esempi illuminanti su come ci si deve comportare, ma per Fénelon il Cristo è inarrivabile nella sua perfezione. Differentemente da quanti lo proponevano come modello da imitare, per Fénelon egli è di fronte a noi come luce, ma il pedagogo deve essere consapevole che, per l'allievo come per tutti noi, egli è un obiettivo che non potrà mai essere raggiunto. Pertanto egli è un modello ideale, questo sì, e in questo senso bisogna parlarne all'allievo, ma sapendo che si tratta di un modello assoluto, al quale non ci si può che avvicinare seguendo i suoi esempi. Noi dobbiamo piuttosto ammirarlo e adorarlo. Con Fénelon si precisa così la distinzione tra modello ed esempio in pedagogia. Fénelon propone esemplarità più quotidiane, accessibili, meno sproporzionate ed angoscianti.Non ha senso indurre sentimenti di insufficienza che ingenererebbero solo sensi di inferiorità, di inadeguatezza e sensi di colpa fuori luogo e sproporzionati. Con gli allievi è necessaria meno severità e più comprensione.

Fénelon è pure distante da Comenio e, come accennavo prima, dalle metodologie didattiche prefissate con rigore seguendo tecniche ritenute di validità universale che permetterebbero di raggiungere obiettivi stabiliti in anticipo o in generale. Egli è 
consapevole, per diretta esperienza, di quanti e quali ostacoli sorgano durante il percorso, e quanti imprevisti si possano incontrare e come possano far insorgere situazioni che appaiono insormontabili. Si possono venire a creare nella dialettica educativa intrichi inestricabili. Fénelon ha compiuto al riguardo esperienze determinanti nell'Istituto per le Nuove Cattoliche dedito alla educazione e " rieducazione » delle figlie di ugonotti.

21 Ma non per questo si deve credere che Fénelon fosse propenso ad assecondare sempre l'allievo fondandosi su un malinteso candore infantile da assolutizzare. D'altronde la teoria dell'innocenza infantile che troviamo già in alcuni autori inglesi, come John Earle o Aphra Behn ${ }^{4}$, ed in seguito anche francesi, può anch'essa portare ad abusi e manipolazioni di chi è più fragile e non ancora formato, e comunque non è presente in Fénelon, pur essendo egli incline ad una pedagogia non autoritaria e non costrittiva. Le due cose non sono necessariamente interdipendenti.

Dalla relazione fatta a Fénelon da Fleury, impegnato con altri precettori (tra cui l'abate di Beaumont, nipote di Fénelon) nell'educazione dei principi, risulta che gli allievi potevano alzarsi, disegnare e anche giocare durante la lezione, per potersi distendere e rilassare, a patto che mantenessero un filo di attenzione sempre desto, in modo che certi argomenti potessero venire ripresi, ripetuti, rivisti riconsiderati alla luce di nuovi raccordi, nuovi punti di vista, nuove utilizzazioni ${ }^{5}$. Per l'educatore è fondamentale osservare i suoi allievi, vederli nella loro realtà più autentica, quando sono rilassati, disinvolti. Questa è la piena e corretta applicazione delle idee pedagogiche di Fénelon.

Nello svolgimento di incombenze o nel rispondere alle richieste, l'allievo che sbaglia deve essere posto in grado di comprendere l'errore, e quindi è assolutamente da evitare che possa essere preso dal terrore di sbagliare e quindi delle conseguenze negative nel giudizio su di lui. L'obiettivo è sempre che sia l'allievo a giungere a convincersi dell'errore compiuto, per fare un salto di qualità in avanti. Mentore nel Télémaque vuole che l'allievo tragga i suoi insegnamenti principalmente dalla vita, dall'esperienza, poiché è la vita che costituisce il reale apprendistato di ciò che si è appreso. L'amico Fleury diceva che ragionamenti troppo astratti allontanano la possibilità di comprensione del senso che la cultura ha per la vita. L'educazione morale mira proprio a far sì che l'allievo faccia ricorso a ciò che ha appreso per imparare sempre più ad avere autonomia di giudizio, nel senso di non lasciarsi sviare dai moti istintivi.

Ne troviamo ulteriore conferma in quel testo sopra menzionato che Fleury scrive il 6 agosto del 1698 per Fénelon, che, pur continuando ad essere gouverneur, da un anno era a Cambrai, per riferirgli a proposito degli studi che egli faceva svolgere agli Infanti di Francia quale sotto-governante dei duchi di Borgogna, Angiò e Berry. Si tratta del Mémoire de ce qui s'est pratiqué dans les estudes des Princes Enfans de France, manoscritto conservato presso gli archivi della British Library di Londra, pubblicato solo nel 1874, e riconosciuto come scritto di Fleury solo nel 1925 da Gaquère ${ }^{6}$.

Già nel 1695 Fénelon, dopo esser stato nominato arcivescovo di Cambrai, aveva dovuto spesso assentarsi, ed aveva scritto a Fleury il programma di studi che avrebbe voluto che fosse seguito per quell'anno e per l'anno successivo ${ }^{7}$; perciò questo Mémoire di Fleury, che costituisce il resoconto di ciò che si è fatto, in un certo senso sembra quasi una risposta alle precedenti sollecitazioni, anche se in realtà era un rapporto completo di tutto il corso degli studi compiuti, includendo anche il periodo iniziale. Comunque da quei due programmi annuali emerge chiaramente la piena sintonia in cui si trovavano $i$ due amici sul piano pedagogico, tanto che Fénelon iniziava il secondo messaggio 
dichiarando il proprio accordo con Fleury e concludeva lasciandogli piena libertà. D'altronde se è pur vero che il titolo di gouverneur non fu ufficialmente mai ritirato a Fénelon, per cui formalmente Fleury restò sempre sous-gouverneur, di fatto almeno dall'agosto $1697^{8}$, quando Fénelon non poté più far ritorno a Versailles, il reale responsabile della educazione degli Enfants de France era divenuto Fleury. I due comunque erano legati da profonda amicizia e fiducia reciproca. Fénelon aveva reso Fleury suo depositario testamentario ; in una occasione aveva anche avuto da lui nel 1691 un prestito in denaro. La loro amicizia si era stretta nel 1684, durante un viaggio a Meaux da Bossuet, e la loro definitiva collaborazione si era cementata durante il periodo in cui furono assieme in missione nelle regioni ugonotte. Quando iniziarono l'educazione degli Enfants de France, il duca di Borgogna, nato il 6 agosto 1682, era il maggiore avendo appena compiuto sette anni, e il duca d'Angiò, Philippe, il futuro re di Spagna, aveva un anno di meno, mentre Charles, il piccolo duca di Berry, ne aveva solamente tre.

Appare subito evidente cosa si intendeva per pedagogia « dolce " e per approccio " gradevole " alle materie di studio se solo si confronta questo corso d'istruzione con quelli allora vigenti ad esempio nei collegi gesuitici. Proprio in quegli anni usciva la famosa opera del padre gesuita Joseph de Jouvancy, De Ratione Discendi et Docendi (1692), sui metodi di studio da seguire nei collegi, da cui possiamo renderci conto della differenza : in primo luogo, per quanto riguarda la frequenza delle pratiche religiose che si esigevano anche dagli allievi più piccoli, e l'importanza che si attribuisce all'osservanza di certe regole nello specifico capitolo " De imbuendis pietate discipuli ", mentre in Fénelon si può constatare la prudente preoccupazione di non eccedere e " annoiare " per timore di essere controproducenti e di " disgustare ») ; differenze rilevanti anche quanto riguarda gli « esercizi della parola », la conoscenza della lingua latina, l'intensità della attività di studio, e certo anche per quel che attiene alla disciplina. $\mathrm{E}$ così per molti altri aspetti, come si può dedurre da un qualsiasi altro testo che riferisca la pratica pedagogica seguita nei collegi, non solo gesuitici, alla fine del Seicento.

Se la sintonia di Fénelon e di Fleury sul piano pedagogico fu completa, Fleury non seguì tuttavia l'amico nelle sue aperte polemiche con Bossuet e soprattutto poi con la corte e con il papato, benché i due amici concordassero su molti punti essenziali anche in materia di educazione religiosa e di politica. La loro intesa sulle modalità non autoritarie, «rilassate », di istruire i propri pupilli, era piena. Anche la loro visione di una pedagogia sostanzialmente indiretta, fondata sulle esperienze, sull'apprendere dalle cose, più che sul nozionismo e l'esercizio della memoria, li portava ad una visione sostanzialmente concorde su moltissimi punti. Era soprattutto importante fornire ai giovani (non solo dunque al futuro sovrano, o alla aristocrazia, ma anche ai semplici borghesi) una formazione storica, in un epoca in cui la totale ignoranza della storia, con la sola eccezione di qualche elemento di storia sacra, e in particolare della storia nazionale moderna, era caratteristica delle classi dirigenti, con l'esclusione di qualche raro erudito o pedante. Nella sua Lettre sur les occupations de l'Académie Françoise, Fénelon indica lo studio della disciplina storica " comme celle qui montre les grans exemples [...], et qui explique par quel chemin les peuples ont passé d'une forme de gouvernement à une autre [et] fait servir les vices mêmes des méchants à l'instruction des bons $»^{9}$. 
Fénelon rispetto all'amico, è indubitabilmente il Telemaco, creazione letteraria di un mondo a parte, fantastico, " provincia pedagogica » dell'immaginario, in cui veniva offerto in esempio al duca di Borgogna un percorso formativo concreto, quotidiano, con tutte le sue difficoltà e complessità. Certo quello del Télémaque è un mondo di fiction, ma in fondo è una realtà rispecchiata, simulata, su cui è più agevole fermarsi a riflettere, a operare rettifiche e correzioni, poiché ci è consentito uscirne per poi farvi ritorno quando vogliamo. In questo Fénelon rinnova radicalmente la tradizione di trattati come gli Specula Principis, poiché il lettore viene invitato ad entrare nello specchio e a vivere con Telemaco la sua esperienza graduale e complessa della vita. La via romanesque ci consente di ritirarci provvisoriamente dalla vita con le sue difficoltà e costrizioni, per poi reintegrarci nella società di appartenenza ma conservando quello spirito critico $\mathrm{e}$ quella libertà di giudizio che la vita narrata ci ha consentito di esercitare. In questo modo Fénelon più che scrivere sul dover essere, sull'infanzia, sulla pedagogia, ci mostra tutto ciò in atto, e soprattutto dà la parola all'allievo Telemaco, per cui l'educando non è più colui di cui si disserta, ma un soggetto che parla, che si fa protagonista del romanzo. Solo più tardi, nel corso del XVIII secolo, vi saranno personaggi infantili o adolescenti come protagonisti ${ }^{11}$. E ciò rappresenta una svolta importante nella storia della letteratura pedagogica in generale. Fénelon ci mostra un allievo che stima ed anzi ama il suo Mentore e che apprende ad esprimersi con linguaggio corretto proprio per esternare le esperienze compiute e le sue riflessioni su di esse in modo appropriato, preciso e comprensibile agli altri. A prima vista Telemaco è per il suo lettore, il duca di Borgogna, il modello da seguire, un modello accessibile, comprensibile, con cui potersi identificare. Anche se ovviamente, ad uno sguardo più attento, il modello proposto, di valore più elevato, è Mentore, ma a questa consapevolezza il lettore giungerà più tardi. Fénelon introduce inoltre il tema del viaggio " guidato » come momento esperienziale di grande importanza formativa. Télémaque è dunque un testo che occupa un posto unico nel contesto della tradizione degli scritti intesi ad usum Delphini pubblicati in quegli anni da Bossuet, da Fleury, da La Mothe La Vayer, o da altri in altri luoghi o anni, non solo perché esso non è un testo contenente un abrégé per l'apprendimento di qualche materia, o consigli o norme per l'educazione di un sovrano, ma in quanto è un romanzo, in cui il protagonista è una sorta di alter ego del destinatario, inaugurando così un genere letterario, quello del romanzo di formazione, " un Bildungsroman ad usum Delphini » (Genette 1982).

30 Questa è la via regia scelta per educare «Telemaco/il duca », questa dunque la modalità che Fénelon indica come " ottimale ». Modalità particolarmente innovativa perché Fénelon non mira alla formazione di un sovrano molto istruito, ma di un sovrano " filosofo ", della filosofia di Mentore, un sovrano soprattutto che operi per il bene 
pubblico più che non per il bene proprio. Un sovrano che potremmo dire illuminato. Anche la visione feneloniana della pedagogia era indubbiamente platonizzante nell'incentrarsi sulla valorizzazione dell'allievo e su una educazione ai valori morali e sulla pratica delle virtù, un'educazione che recupera i classici greci e latini, in quanto essi hanno moltissimo da darci sul piano dell'humanitas, anche se essi vanno riletti e riproposti con quella traslazione che consenta loro di essere rivivificati.

31 Fénelon fu comunque letto, e quindi « sentito » variamente : i philosophes ne furono entusiasti per la sua battaglia contro l'assolutismo, fu adorato da Rousseau (tanto che per molto tempo lo si è interpretato solo come suo ispiratore e quindi precursore), fu in gran voga nel periodo repubblicano, e continuò a lungo ad esser visto solo come spirito libero, o antesignano di tutti gli spiriti liberi. Per Flaubert invece, dato che nel Télémaque Fénelon parla per interposta bocca di Omero, il risultato non poteva essere che qualcosa di artificioso, "stupido e falso da tutti i punti di vista ». Oppure fu letto alla luce del conflitto di Fénelon con il « Padre ", il grande Bossuet, o della supposta soggezione filiale alla "Madre ", Madame Guyon, da cui sarebbe stato plagiato. In effetti la sua personalità originale è stata dalla storiografia in certi casi inficiata, e la sua personale propensione ad una teologia mistica è stata considerata a volte quasi secondaria, almeno da parte degli studiosi di storia della pedagogia.

Benché Fénelon non avesse conosciuto mai, a quanto pare, l'esperienza di stati estatici, il misticismo è certamente parte integrante del suo più profondo sentire. Percepiva come un incolmabile abisso la distanza tra le possibilità della ragione umana e la parole de Dieu che la trascende ed è irraggiungibile. Senonché questo suo sguardo, che il silenzio e la concentrazione richieste dalla preghiera riescono a far sporgere verso le infinite profondità dell'abisso, attraggono la mente umana sino al punto da incitarla a gettarvisi con tutto l'abbandono, e con amore puro (cf. Varillon 1957 e Perrotti 1994). E comunque se il linguaggio scritto, sia pure quello della più sublime delle poesie, non può rendere l'esperienza della vicinanza, o addirittura dell'intimità, del colloquio che nella meditazione si può instaurare con la voce divina, non resta che porre come obiettivo sublime l'ascesi spirituale e come obiettivi intermedi l'affinamento della sensibilità, il gusto del bello in ogni forma espressiva, l'attrazione verso modelli esemplari di pratica della virtù, al centro del proprio pensiero religioso, filosofico e pedagogico.

Quindi nei suoi scritti, almeno per quanto riguarda l'educazione, Fénelon non formula una specifica teoria, né dà vita ad una visionaria utopia, ma crea un grande romanzo di formazione, inaugurando una lunga e fiorente stagione. Vengono alla luce importanti concetti pedagogici, in parte innovativi, che esprimono l'esigenza di un moderato, ma non per questo meno incisivo, rinnovamento di carattere sociale, morale, politico, oltre che educativo. Un'educazione "indiretta ", un nuovo modo di vedere il rapporto maestro-allievo, basato sul dialogo tra un mentore e il suo giovane partner verso cui il primo nutre amore filiale, il valore attribuito all'esperienza, che è il mentore stesso a sollecitare nell'allievo, il modello classico di un regime di vita semplice e lontana dai lussi, diventano in effetti strumenti di critica della società e della mentalità del tempo. Nel e col romanzo si delinea una concezione pedagogica che fa da supporto a tutto il libro (confermata tra le righe in tutti gli scritti), che scaturisce da esemplificazioni di un percorso formativo, con espliciti ripensamenti, errori, ritocchi resisi indispensabili, simulazioni. 

centrale l'attenzione ai valori morali, soprattutto nelle opere per il duca di Borgogna, possibile futuro re. Nell'esercizio del comando supremo, si evidencia soprattutto la sacralità della figura regia, e dunque è per Fénelon ancor più necessario, se possibile, educare alla moderazione, alla moralità, coltivare la spiritualità. Fénelon valorizza dunque la formazione culturale ampia come una via verso la pratica della virtù, della prudenza, e della saggezza. E pertanto ritiene ancor più pericoloso lasciare un principe ereditario in balia di influssi mondani deleteri, come troppo spesso accadeva ed era accaduto in molteplici casi. Fénelon non incentra la sua educazione sull'obiettivo di educare all'eloquenza persuasiva, anche se tocca i temi dell'arte retorica. Così come certo a Fénelon non interessa educare alla magnificenza e all'apparenza (aspetto tuttavia notoriamente tanto curato e non solo in quell'epoca, e non solo nell'educazione principesca), quanto alla sostanza soprattutto dei valori spirituali, in vista proprio di ciò che sarà richiesto dall'alta funzione etica che deve sentirsi chiamato a svolgere un sovrano nel suo ruolo regale. La sua concezione complessiva assomiglia poco dunque alla educazione principesca corrente come a quella praticata anche nei migliori casi. Per Fénelon « principesca » deve significare « la migliore », o piuttosto quella ottimale (e dunque per questo esemplare anche per altri allievi siano essi nobili o meno ${ }^{12}$. Deve essere tale poiché nell'« utopia » feneloniana il sovrano in quanto supremo legislatore ha il potere demiurgico di forgiare il suo popolo e dunque deve avere assimilato, introiettato e fatti propri, i valori più puri di giustizia, equanimità, clemenza, moralità, ecc. Sul successo nella educazione che si dà al futuro re, si gioca una partita dalla posta molto alta, e di interesse generale. Certo si potrebbe anche dire che Fénelon ebbe la buona sorte di avere sotto le sue cure il duca di Borgogna ${ }^{13}$. Tuttavia è senza dubbio alle qualità personali di Fénelon, all'insieme delle sue scelte complessive e particolari, e alla sua determinazione nel perseguire gli obiettivi preposti, che dobbiamo l'esemplarità del percorso formativo praticato col duca di Borgogna di cui ancor oggi parliamo e su cui riflettiamo. Ed essa è tale perché nel modo di farsi Mentore/Minerva da parte di Fénelon, troviamo un modello esemplare per leggere e interpretare una visione della formazione dell'uomo. Ed infine perché nell'insieme dell'opera feneloniana ci risulta possibile individuare esigenze e problematiche di una cultura che richiama con insistenza all'esperienza concreta, alla conduzione di una vita consapevole e, in definitiva, alla necessità di acquisire la capacità di una interpretazione critica del proprio mondo, meno per respingerlo, quanto piuttosto per tentare di migliorarlo.

\section{Bibliographie}

BARNARD, Howard Clive (1966), Fénelon on Education, Cambridge, University Press.

BERGAMO, Mino (1991), L'anatomia dell'anima. Da François de Sales a Fénelon, Bologna, Il Mulino. 
Correspondance générale de Fénelon (1827), Paris, Jeune.

DANIEL-ROPS, Henri (1987), « Fénelon », in Dizionario dei capolavori, Torino, UTET.

FÉNELON (1843), OEuvres diverses, Paris-Lyon, Librairie Catholique/Perisse Frères.

------(1996), L'amore disarmato, a cura di B. Papasogli, Milano, Ediz. Paoline.

------(2002), Spiegazione delle massime dei Santi sulla vita interiore, a cura di Marco Vannini, Milano, edizioni San Paolo.

FÉNELON / FLEURY (1874), «Plan des Études de Monseigneur le Duc de Bourgogne », Annuaire Bulletin de la Société de l'Histoire de France, XI, 54-60.

FERRARI, Monica (1996), La paideïa del sovrano. Ideologie e strategie nell'educazione principesca del Seicento, Firenze, La Nuova Italia editrice.

FLEURY, Abbé Claude (1681), Les Moeurs des Israélites, où l'on voit le modèle d'une politique simple \& sincère pour le gouvernement des États \& de la réformation des moeurs, Paris, Pierre Aubouyn, etc.

-----(1714), Portrait de Louis, Duc de Bourgogne, puis Dauphin, néle 6 d'aoust 1682, mort le 18 février 1712, à Paris.

FOUCAULT, Michel (1976), La volonté de savoir, Paris, Gallimard.

GAQUÈRE, François (1925), La vie et les oeuvres de l'abbé Claude Fleury, Paris, J. De Gigord.

GENETTE, Gérard (1982), Palimpsestes. La littérature au second degré, Paris, Seuil.

GOUHIER, Henri (1969), Fénelon et la prédication, Paris, Klincksieck.

-----(1977), Fénelon philosophe, Paris, Vrin.

HILLENAAR, Henk (1984), « Inconscient et religion dans Télémaque », in La pensée religieuse dans la littérature et la civilisation du XVII ${ }^{e}$ siècle en France, Seattle, Papers on French 17th Century Litterature.

JOLIBERT, Bernard (1977), L'enfance au 17e siècle, Paris, Vrin.

NICOLE, Pierre (1670), De l'éducation d'un prince, ed. in italiano : Dell'educazione di un principe, a cura di C.A. Sacheli, Milano, 1915.

OPPICI, Patrizia (1986), Bambini d'inchiostro. Personaggi infantili e sensibilité nella letteratura francese, Pisa, Editrice Goliardica.

PANCERA, Carlo (1987), « L'insegnamento della storia in Francia tra la metà del XVII e la metà del XVIII secolo ", in M. Miegge (dir.), Le parole della storia.Saggi sulle rappresentazioni del tempo tra riforma e illuminismo, Bologna, Cappelli editore.

-----(1991), Il pensiero educativo di Fénelon, Firenze, La Nuova Italia editrice, Appendice IV : 142-145.

------(1996), « L'originalità del pensiero educativo di Claude Fleury », Annali di Storia dell'Educazione e delle Istituzioni Scolastiche, n. 3, Brescia, La Scuola editrice, 149-182.

------(2002), « Claude Fleury e l'educazione dei piccoli principi di Condé, e dei tre principini les Enfants de France ", in La formazione del Principe in Europa dal Quattrocento al Seicento. Un tema al crocevia di diverse storie, Convegno internazionale diretto da P. Carile, Ferrara 19-20 aprile 2002, in corso di stampa.

PERROTTI, Gabriele (1994), Il tempo e l'amore. Metafisica e spiritualità in Fénelon, Napoli, Bibliopolis. SPENCER, Jane (1986), The Rise of the Woman Novelist, Oxford, Blackwell. 
VARILLON, François (1957), Fénelon et le pur amour, Paris, Seuil.

WANNER, Raymond E. (1975), Claude Fleury as an educational historiographer and thinker, The Hague, Martinus Nijhoff.

\section{NOTES}

1. A questo proposito ben giunge l'edizione in italiano della sua Spiegazione delle massime dei Santi sulla vita interiore, opera curata da Marco Vannini (Fénelon 2002).

2. Piuttosto uno specchio d'acqua calmo e quindi superficialmente fermo, ma al contempo liquido, pieno di vita, e di potenzialità. Si vedano le espressioni ricorrenti nel carteggio epistolare, e sottolineate da Benedetta Papasogli nella sua bella antologia in italiano di 70 lettere (Fénelon 1996). Cf. anche Hillenaar 1984.

3. Cf. Bergamo 1991 ; tra l'altro Carlo Cordié definì Fénelon « un filiale lettore di François de Sales » (Cultura e scuola, n. 125, 1993), anche Henri Daniel-Rops trova nella corrispondenza « il tono così saggio e umano di François de Sales » (1987).

4. Aphra Behn, la prima scrittrice di professione, che tradusse in inglese Fontenelle, autrice di commedie molto popolari sotto Carlo II, scriveva nel 1688 : «Dio crea tutte le cose buone : l'uomo ci mette le mani, ed ecco esse divengono corrotte ", con tipico accento moralistico puritano. Cf. Spencer 1986.

5. Si veda il testo del Mémoire scritto da Fleury a Fénelon, da me tradotto e riportato in : Pancera 1996 (ora riprodotto anche in Pancera 2002. Cf. anche note successive 6 e 7.

6. È conservato alla British Library di Londra nelle « Lettres et Pièces diverses de Fénelon » tra gli Egerton manuscripts, 36, ff. 11-13. È stato pubblicato come testo di Fénelon e/o di Fleury, col titolo «Plan des Études de Monseigneur le Duc de Bourgogne » (Fénelon / Fleury 1874). Ed è stato segnalato tra le opere di Fleury da François Gaquère (1925). Una trentina d'anni fa è stato riportato integralmente in appendice al proprio lavoro su Fleury, dallo storico americano Raymond E. Wanner (1975), che lo ha anche tradotto in inglese.

7. Cf. Correspondance générale de Fénelon (1827 : III, 354-357). Su quelle lettere si veda anche : Barnard 1966. (Il testo dei due programmi di studio è da me riportato e tradotto in italiano nell'Appendice IV in Pancera 1991). Si veda anche : Mémoire sur l'éducation des ducs de Bourgogne, d'Anjou et de Berry, rédigé en 1696 par le marquis de Louville, in : Fénelon 1843 : IV, 82).

8. Per una visione generale dell'opera precettoriale di Fénelon e Fleury (ma anche di Bossuet e di La Mothe Le Vayer con il Gran Delfino), cf. Ferrari 1996 : $72-87$.

9. Dal § VIII. Sul contesto di queste proposte, e sull'importanza di Bossuet in questo campo, mi si consenta di rinviare al mio contributo (Pancera 1987, capitolo IV).

10. Cf. Fleury 1681, cui faranno seguito Les Moeurs des Chrétiens, Catéchisme Historique, etc.

11. Cf. Oppici 1986, anche se verte piuttosto sul secondo Settecento.

12. Concetto che d'altronde aveva già ben definito Pierre Nicole (1670).

13. Si veda il suo ritratto per le parti in cui è descritto quale allievo studioso in Fleury 1714.

\section{RÉSUMÉS}

Absolutism, tolerance, education, pedagogy, preceptor, Fénelon, Fleury 
Fénelon puts forward his anti-absolutist thought within an organic conception of man and society based on tolerance. From grammar to history, from language to behaviour, form the formation of the taste for reading to the analysis of inner necessities, Fénelon's work develops into an all encompassing pedagogical itinerary and a non-authoritarian intervention stricly in keeping with the ideal of a prince capable of retaining moral and spiritual values. We also pointed out that the intellectual interchange between Fénelon and Fleury was so intense that very often it is hard to tell their contributions about formation apart.

Fénelon précise sa pensée anti-absolutiste dans une conception organique de l'homme et de la société basée sur la tolérance. De la grammaire à l'histoire, du langage au comportement, de la formation du goût de la lecture à l'examen des exigences intérieures, se compose un itinéraire formatif et une modalité d'intervention non-directive cohérente avec l'idéal d'un prince capable d'être en harmonie avec les valeurs éthiques et spirituelles. On a également souligné que les échanges intellectuels entre Fénelon et Fleury ont été si intenses que souvent les différentes contributions des deux précepteurs ne sont pas séparables, du moins dans le domaine de la formation.

INDEX

Mots-clés : absolutisme, tolérance, éducation, pédagogie, précepteur, Fénelon, Fleury

\section{AUTEUR}

\section{CARLO PANCERA}

Università di Ferrara 